\title{
Prognostic Implications of Q Waves and T-Wave Inversion Associated With Early Repolarization
}

\author{
Abhimanyu Uberoi, MD, MS; Karim Sallam, MD; Marco Perez, MD; Nikhil A. Jain, BA; \\ Euan Ashley, MRCP, DPhil; and Victor Froelicher, MD
}

\begin{abstract}
Objective: To evaluate the prevalence of early polarization (ER) in a stable population and to evaluate the prognostic significance of the association or absence of $\mathrm{Q}$ waves or T-wave inversion (TWI).

Patients and Methods: In this retrospective study performed at the university-affiliated Palo Alto Veterans Affairs Health Care Center from March 1, 1987, through December 31, 1999, we evaluated outpatient electrocardiograms. Vital status and cause of death were determined in all patients, with a mean \pm SD follow-up of $7.6 \pm 3.8$ years.

Results: Of the 29,281 patients, $87 \%$ were men and 13\% were African American. Inferior or lateral ER was present in 664 patients (2.3\%): in inferior leads in 185 (0.6\%), in lateral leads in 479 (1.6\%), and in both inferior and lateral leads in $163(0.6 \%)$. Only when Q waves or TWI accompanied ER was there an increased risk of cardiovascular death (Cox proportional hazards regression model, 5.0; $95 \%$ confidence interval, 3.4-7.2; $P<.001$ ).

Conclusion: Common patterns of ER without concomitant $Q$ waves or TWI are not associated with increased risk of cardiovascular death; however, when either occurs with ER, there is a hazard ratio of 5.0. These findings confirm that ER is a benign entity; however, the presence of $Q$ waves or TWI with ER is predictive of increased cardiovascular death.
\end{abstract}

๑ 2012 Mayo Foundation for Medical Education and Research Mayo Clin Proc. 2012;87(7):614-619

\section{For editorial comment, see page 610}

From the Department of Medicine (A.U.) and the Division of Cardiovascular Medicine, Department of Medicine (K.S., M.P., E.A., V.F.), Stanford University School of Medicine, Stanford, CA; College of Arts and Sciences, Washington University, St. Louis, MO (N.A.J.); and Cardiology Section, Veterans Affairs Palo Alto Health Care System, Palo Alto, CA (V.F.).

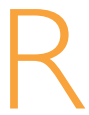

ecent studies have suggested an association between early repolarization (ER) and cardiovascular death. Early repolarization has a reported prevalence as high as 5\% and in many affected patients is asymptomatic and identified in the ambulatory setting. The seminal paper by Haissaguerre et $\mathrm{al}^{1}$ was based on a unique population of 206 patients with idiopathic ventricular fibrillation. A community epidemiologic study subsequently found ER in the inferior leads to have a modest adjusted hazard ratio (HR) for cardiac death. ${ }^{2}$ These 2 studies counter the data supporting the benign nature of ER in healthy athletes and other apparently healthy individuals. ${ }^{3}$ Thus, delineating a risk associated with ER would carry important clinical practice considerations for practitioners. In a previous study, we found no risk associated with any component of ER. ${ }^{4}$ To resolve this controversy, we evaluated the association of ER with diagnostic Q waves and T-wave inversion (TWI) and prognosis.

\section{PATIENTS AND METHODS}

\section{Clinical Population}

We performed a retrospective study of 45,829 inpatient and outpatient electrocardiograms (ECGs) from March 1, 1987, through December 31, 1999 , at the Veterans Affairs (VA) Palo Alto Health Care System. Since 1987, this VA facility has used a cen- tralized computerized ECG system for collection, storage, and analysis of ECGs (GE Healthcare, Wauwatosa, WI). This system has been approved by the US Food and Drug Administration and the European Union, and is used widely around the world. All patients were seen at the main VA facility or its satellite clinics, and ECGs were ordered by health care professionals for clinical indications. We considered the first ECG and excluded ECGs with one or more of the following: inpatient status $(n=12,319)$, atrial fibrillation or flutter $(n=1253)$, ventricular rate $>100$ beats/min $(n=2799)$, QRS duration $>120 \mathrm{~ms} \quad(\mathrm{n}=3141)$, paced rhythm $(n=290)$, ventricular preexcitation $(n=42)$, and acute myocardial infarction $(n=29)$. Thus, 29,281 ECGs were included in the study.

\section{ECG Interpretation}

The ST level was computer measured at the end of the QRS complex, and ER was considered as elevation of $1 \mathrm{~mm}$ or more $(\geq 0.1 \mathrm{mV})$ at the end of the QRS complex when compared with the isoelectric PR segment. J waves were defined as positive deflections occurring at the QRS-ST segment junction. Slurring was defined as a conduction delay in the QRS downslope (Figure 1). Two criteria were used to interpret ECGs with ER, including the presence of either (1) adjacent (2 contiguous) leads in any area lead group (inferior: II, III, and aVF; lateral: I, aVL, 
and $\mathrm{V}_{4}$ through $\mathrm{V}_{6}$; and anterior: $\mathrm{V}_{1}$ through $\mathrm{V}_{3}$ ) or (2) only 1 lead, or "any lead," with ER in the area. Anterior lead ST elevation was considered separately because of its presence in the Brugada pattern and lack of $\mathrm{J}$ waves or slurring. The measurements and criteria for diagnostic $Q$ waves were those used by the GE 12SL ECG program (http:// www.gehealthcare.com/euen/cardiology/products/ diagnostic_ecg/algorithms/index.html), and TWI was coded as less than $0.5 \mathrm{~mm}(0.05 \mathrm{mV})$. All ECGs coded as having ER were visually inspected and coded by 3 blinded investigators (A.U., M.P., V.F.) for the presence of $J$ waves or slurring because these are not recognized by the computer program.

\section{Outcomes}

The primary outcome variable was time to cardiovascular death. The California Health Department Service and the Social Security Death Index were used to ascertain the vital status of each patient as of December 31, 2002. Accuracy of cause of death was reviewed by 2 clinicians (A.U., V.F.) blinded to the ECG results and were confirmed using VA computerized medical records.

\section{Statistical Analyses}

All statistical analyses were performed using commercially available software: NCSS version 2007 (NCSS, LLC, Kayesville, UT) and SAS version 9.1 (SAS Institute, Inc, Cary, NC). Unpaired $t$ tests were used for comparisons of continuous variables, and $\chi^{2}$ tests were used to compare dichotomous variables between patient groups. Survival analysis was performed using the Kaplan-Meier method. Cox proportional HRs were used to determine associations between ST-elevation patterns and outcomes. Analyses were adjusted for age, sex, race/ ethnicity, Minnesota ECG criteria for coronary disease, ${ }^{5}$ and heart rate. The proportional hazards assumption was confirmed using the scaled Schoenfeld residuals.

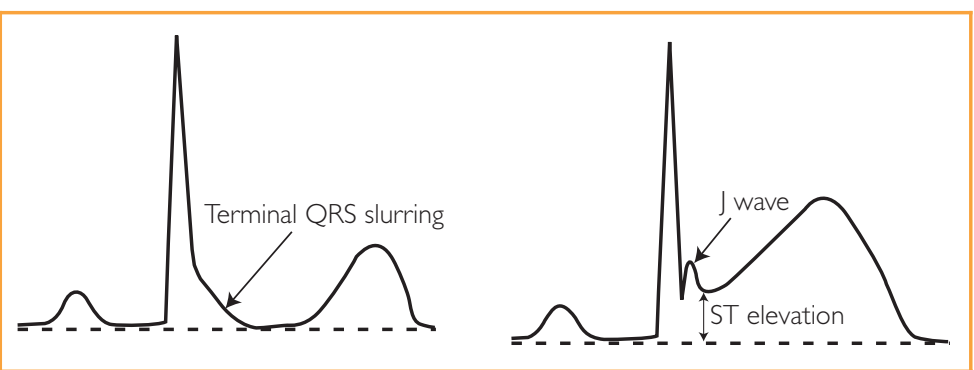

FIGURE 1. Definitions of electrocardiographic findings.

\section{RESULTS}

Of the 29,281 patients, 25,544 (87.2\%) were men (mean \pm SD age, $55 \pm 14$ years) and 3737 (12.8\%) were women ( $56 \pm 17$ years). The population was differentiated into 2 categories: African Americans (13\%) and whites including Hispanics (87\%). There were 6739 deaths (1995 cardiovascular) over a follow-up of $7.6 \pm 3.8$ years. Visual inspection of all ECGs coded as having ER via computer measurement revealed that isolated J waves or slurring were the major cause ( 90\%) in the inferior leads, whereas the converse was true in the lateral leads, in which ST elevation predominated, although usually accompanied by J waves or slurring.

Table 1 gives the demographic characteristics of the stable outpatient population. Figure 2 demonstrates the prevalence of ER by age in any lead or adjacent leads in men and women. Table 2 gives the association of ER using the "any lead" in an area criterion with the presence of diagnostic Q waves and TWI in the same area, and cardiovascular death. This table demonstrates that the cardiovascular deaths were clustered in patients with ECGs with diagnostic Q waves and TWI within the patterns of ER. In other words, in patients with ECGs with ER without $\mathrm{Q}$ waves or TWI, there were comparatively fewer cardiovascular deaths (range, 3.2\%-10\%) than in those with ER and these abnormalities

\begin{tabular}{|c|c|c|c|}
\hline Variable & Men $(n=25,544[87 \%])$ & Women $(n=3737[13 \%])$ & $P$ value \\
\hline Age $(y)$ & $55 \pm 14$ & $56 \pm 17$ & $<.01$ \\
\hline \multicolumn{4}{|l|}{ Race/ethnicity, No. (\%) } \\
\hline White/other & $21,990(86)$ & 3406 (9I) & $<.01$ \\
\hline African American & $3554(14)$ & $331(9)$ & $<.01$ \\
\hline Weight (Ib) & $187 \pm 39$ & $|5| \pm 36$ & $<.01$ \\
\hline Height (inch) & $69 \pm 3$ & $64 \pm 3$ & $<.01$ \\
\hline Body mass index $\left(\mathrm{kg} / \mathrm{m}^{2}\right)$ & $27 \pm 5$ & $26 \pm 6$ & $<.01$ \\
\hline Heart rate (beats/min) & $71 \pm 13$ & $69 \pm 11$ & $<.01$ \\
\hline
\end{tabular}




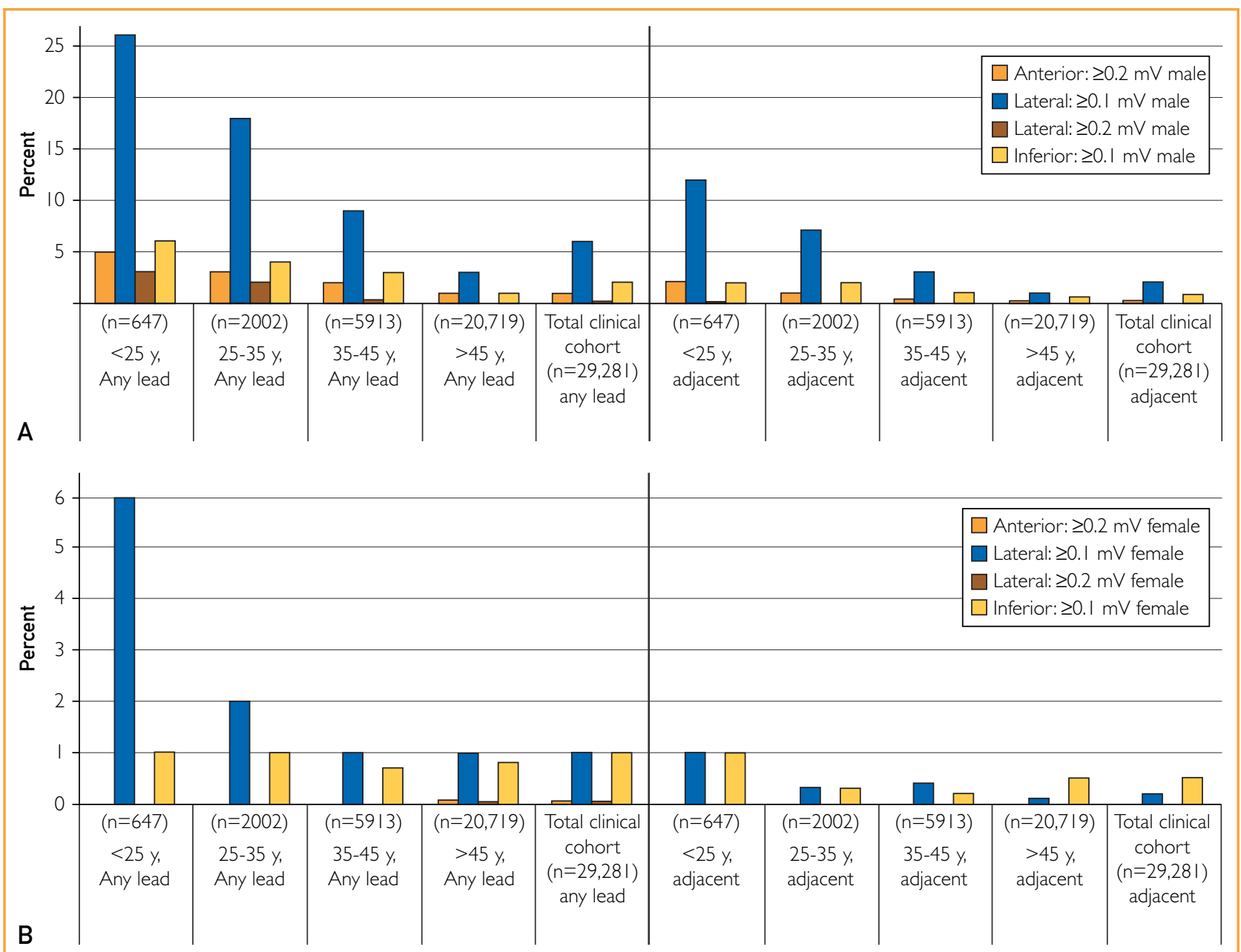

FIGURE 2. ST elevation by age in any lead or adjacent leads in men (A) and women (B).

(range, 20\%-45\%). Figure 3, A presents the KaplanMeyer survival curve for the cohort with ER without TWI or $\mathrm{Q}$ waves $(\mathrm{n}=1665)$ compared with all others in the population without the ECG finding $(n=27,616)$. Figure 3, B presents the Kaplan-Meyer survival curves for the cohort with ER and TWI or diagnostic $Q$ waves $(n=114)$ compared with the rest of the population who did not meet the ECG criteria $(n=29,167)$. The presence of ER in any lead does not affect survival; however, when concomitant with $\mathrm{Q}$ waves or inverted $\mathrm{T}$ waves, there is an association with decreased survival.

Table 3 gives the unadjusted and adjusted Cox proportional hazards results in the 3 surface areas of the heart for the major ER patterns, diagnostic $Q$ waves, and TWI in the entire clinical cohort. Early repolarization in the lateral leads was associated with halving the risk of cardiovascular death; however, this advantage was no longer present after ad- justing for age and sex (ie, the decreased mortality occurred because the pattern was more prevalent in young men). When adjusted only for TWI or diagnostic $\mathrm{Q}$ waves in lateral leads, the decreased risk of death remained, and there was significant risk associated with both abnormalities. ST elevation in the anterior or inferior leads was not univariately associated with cardiovascular death. Adjustment for age and sex found a significant risk for anterior ST elevation that was no longer present when adjusted for anterior Q waves and TWI. Therefore, the risk of death was due to these abnormalities and not to the anterior ST elevation itself. Adjustment for age and sex supports a small significant risk for inferior ER; however, no significant risk was present after adjustment for inferior Q waves and TWI. Similarly, the risk was due to Q waves and TWI and not to the inferior ER itself. Early polarization in any area accompanied by Q waves or TWI in the same area was 


\begin{tabular}{|c|c|c|c|}
\hline Variable & $\begin{array}{c}\geq 0.2 \mathrm{mV} \text { ST elevation } \\
\text { in any anterior lead }\end{array}$ & $\begin{array}{l}\geq 0.1 \mathrm{mV} \text { ST elevation } \\
\text { in any inferior lead }\end{array}$ & $\begin{array}{l}\geq 0.1 \mathrm{mV} \text { ST elevation } \\
\text { in any lateral lead }\end{array}$ \\
\hline Total & 326 (33 CVD, 10\%) & 485 (3। CVD, 6.4\%) & |48| (53 CVD, 3.8\%) \\
\hline No Q waves or TWI & 301 (24 CVD, 8\%) & 424 (27 CVD, 6.4\%) & I 447 (45 CVD, 3.2\%) \\
\hline TWI $\geq 0.05 \mathrm{mV}$ in the area & 5 (I CVD, 20\%) & 19 (4 CVD, 21\%) & 30 (7 CVD, 23\%) \\
\hline Diagnostic $Q$ waves in the area & 21 (9 CVD, 43\%) & 52 (II CVD, 2I\%) & 8 (2 CVD, 25\%) \\
\hline Both TWI and $\mathrm{Q}$ waves in the area & I (I CVD, I00\%) & 10 (2 CVD, 20\%) & 4 (I CVD, 25\%) \\
\hline
\end{tabular}

associated with the greatest risk of cardiovascular death of any ECG pattern studied. This trend persisted despite adjustment for age and sex. When adjusted for African American race along with age and sex, the risk remained (mean [range] HR, 3.4 [2.4-5.0]; $P<.001)$. Consistent with findings of previous studies, both diagnostic $Q$ waves and TWI were associated with significant risk; however, the combination with ER generated the greatest risk.

\section{DISCUSSION}

To our knowledge, this is the largest, multiple race/ ethnicity, long-term study of the association among ER, Q waves, TWI, and cardiovascular death. Our results in a stable ambulatory population demonstrate that ER patterns do not increase the risk of cardiovascular death. When concomitant $\mathrm{Q}$ waves or TWI are present, however, a greater risk is present than when Q waves or TWI are present alone. The risk was consistent across all lead groups. Conversely, ER in the absence of Q waves or TWI in any lead groups was not associated with increased cardiovascular death. Indeed, ER alone conferred a lower risk of cardiovascular death, in particular whenever the lateral leads were involved.

Our results diverge from data from recent prognostic studies of ER. Sinner et $\mathrm{al}^{6}$ documented an increased HR of death associated with ER, in particular in the inferior leads. A major limitation of their study, however, was the use of a case-cohort design, which considered only a subset of their communitybased population "enriched by all those who died," resulting in a "limited challenge." The study by Tikkannen et $\mathrm{al}^{2}$ was limited by noncomputerized ECG acquisition; the paper ECG recordings from more than 30 years ago required using the "adjacent lead" criteria for accuracy, unlike today's ECG analyses, which rely on waveforms averaged over 10 seconds. Furthermore, the authors failed to specifically control for the presence of Q waves and TWI. ${ }^{2}$ A third outcome study, by Haruta et al, ${ }^{7}$ concluded that ER was predictive only of "unexplained death." Al- though unexplained death was intended to be a surrogate for cardiac arrest, the authors' definition inadvertently included any death of unexpected cause and any unexplained accidental death. Furthermore, the relatively low incidence of unexplained death and lack of an HR for cardiovascular death place their conclusions in question.

Beyond data acquisition and definition of ER, there are other reasons why our results conflict with those of previous studies. First, the demographic

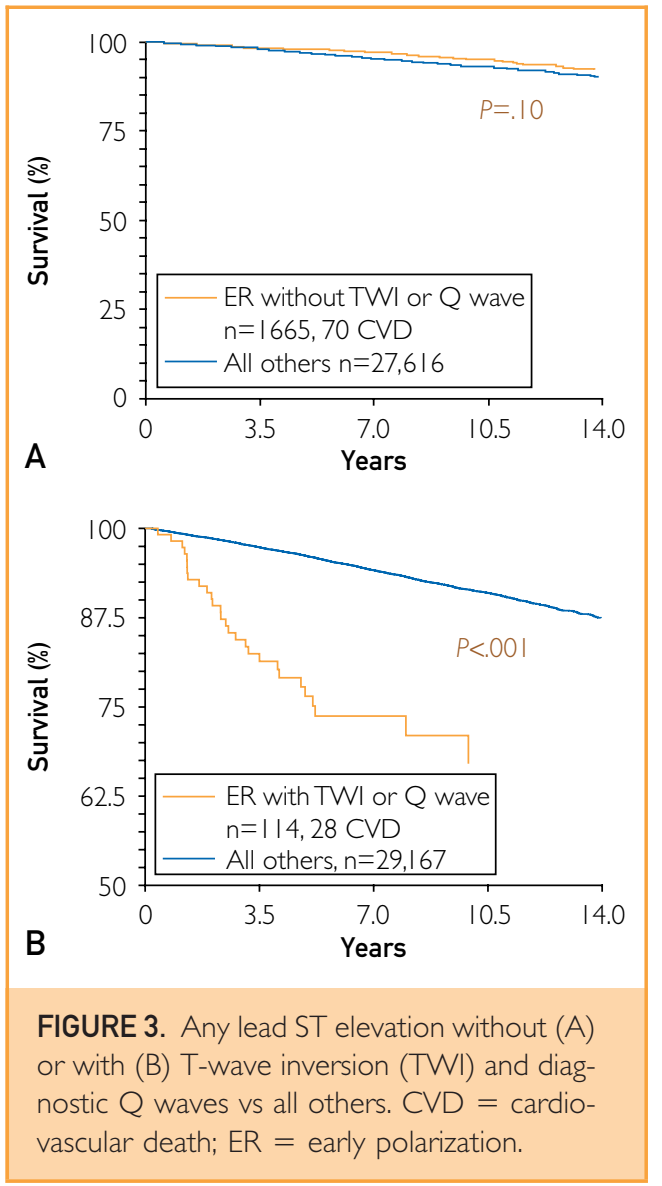


TABLE 3. Unadjusted and Adjusted Cox Hazard Results in the 3 Surface Areas of the Heart for the Major ST-Elevation Patterns, Diagnostic Q Waves, and TWI in the Entire Clinical Cohort ${ }^{\text {a.b }}$

\begin{tabular}{|c|c|c|c|c|c|c|}
\hline \multirow[b]{2}{*}{ Cardiovascular death } & \multicolumn{6}{|c|}{ Hazard ratio ${ }^{c}$} \\
\hline & Univariate analysis & $P$ value & $\begin{array}{l}\text { Adjusted for } \\
\text { age and sex }\end{array}$ & $P$ value & $\begin{array}{l}\text { Adjusted for ECG } \\
\text { abnormality }^{d}\end{array}$ & $P$ value \\
\hline $\begin{array}{l}\text { Anterior lead ST elevation }\left(V_{1}, V_{2}, V_{3}\right) \text { using } \\
0.2-m V \text { criteria }\end{array}$ & $1.4(1.0-2.0)$ & .50 & $2.0(1.4-2.8)$ & $<.001$ & $1.4(1.0-1.9)$ & .8 \\
\hline Anterior lead TWI $\left(V_{2}, V_{3}\right)$ & $2.0(1.5-2.7)$ & $<.001$ & $1.6(1.2-2.2)$ & .004 & NA & NA \\
\hline Anterior diagnostic $\mathrm{Q}$ wave & $3.0(2.5-3.7)$ & $<.001$ & $2.1(1.7-2.6)$ & $<.001$ & NA & NA \\
\hline $\begin{array}{l}\left.\text { Lateral lead ST elevation ( } \mathrm{I}, \mathrm{aVL}, \mathrm{V}_{4}, \mathrm{~V}_{5}, \mathrm{~V}_{6}\right) \\
\text { using } 0.1-\mathrm{mV} \text { criteria }\end{array}$ & $0.5(0.3-0.6)$ & $<.001$ & $0.9(0.3-0.6)$ & .6 & $0.5(0.4-0.6)$ & $<.001$ \\
\hline Lateral lead TWI & $4.4(3.9-5.0)$ & $<.001$ & $2.6(2.3-3.0)$ & $<.001$ & NA & NA \\
\hline Lateral diagnostic $\mathrm{Q}$ waves & $2.4(1.6-3.7)$ & $<.001$ & $1.6(1.0-2.5)$ & .03 & NA & NA \\
\hline $\begin{array}{l}\text { Inferior lead ST elevation (II, III, aVF) using } \\
\text { 0.I-mV criteria }\end{array}$ & $1.0(0.7-1.4)$ & .9 & $1.6(1.1-2.2)$ & .01 & $1.0(0.7-1.4)$ & .8 \\
\hline Inferior lead TWI (aVF, II) & $2.8(2.4-3.3)$ & $<.001$ & $2.2(1.9-2.6)$ & $<.001$ & NA & NA \\
\hline Inferior diagnostic $\mathrm{Q}$ waves & $3.0(2.7-3.4)$ & $<.001$ & $2.0(1.8-2.3)$ & $<.001$ & NA & NA \\
\hline $\begin{array}{l}\text { ST elevation in any area accompanied by } \\
\text { TWI or Q waves in the same area }\end{array}$ & $5.0(3.4-7.2)$ & $<.001$ & $3.4(2.4-5.0)$ & $<.001$ & NA & NA \\
\hline $\begin{array}{l}{ }^{\mathrm{a}} \mathrm{NA}=\text { not applicable; } \mathrm{TWI}=\mathrm{T} \text {-wave inversion. } \\
\text { b Values are presented as mean (range). } \\
{ }^{\mathrm{c} C o x} \text { hazard regression hazard ratio. } \\
{ }^{\mathrm{d}} \text { Heart rate, TWI, and/or diagnostic Q waves in th }\end{array}$ & area & & & & & \\
\hline
\end{tabular}

characteristics in our study were different from those of previous studies. Our study population was older, included a higher percentage of men, and 13\% were African American as opposed to the ethnically homogeneous northern European and Asian populations studied by other groups. ${ }^{2,6,7}$ Second, only Tikkanen et $\mathrm{al}^{2}$ adjusted their hazards models for concomitant ECG abnormalities, as we did. By adjusting our HR for 2 of these findings independently, we appreciated a complex interaction between these phenomena and ER, namely, that the presence of ER predicted a higher HR than would be predicted by $\mathrm{Q}$ waves or TWI alone. Tikkanen et $\mathrm{al}^{2}$ defined an HR for inferior R-wave downslope abnormalities that remained significant after adjustment; however, they used a composite abnormal ECG variable that included ST abnormalities and bundle branch block. This disagreement may speak to the variable interaction that various ST abnormalities have with concomitant ER.

Several studies, including those mentioned, have reported that there is clear heterogeneity in the risk associated with various ER patterns. Tikkanen et $\mathrm{al}^{8}$ concluded that, in their population data, some ST-segment slopes combined with J waves predicted a small risk of death due to arrhythmia, whereas some predicted no risk at all. Furthermore, Rosso et $\mathrm{al}^{9}$ found that horizontal or downsloping ST seg- ments in association with J waves were associated with an odds ratio of 4 for idiopathic ventricular fibrillation when compared with matched controls, whereas upsloping ST segments carried no increased risk. This highlights the marked heterogeneity in defining ER and the definite differences in risk predicted by each of the patterns. Clinically, this would necessitate that methods be carefully spelled out and terminology be clear. ${ }^{10}$

It is important to note that none of the population studies identified an increased HR associated with isolated ST elevation in any lead distribution. ST elevation is the exclusive feature used by computerized ECG machines to generate the analysis statement "Early Repolarization" at the top of the ECG printout with no ability to recognize J waves or slurring. ${ }^{11-14}$ These clinical statements have been repeatedly found to influence clinical decision making and, hence, carry considerable influence in dictating management. ${ }^{15-17}$ Thus, one can imagine the erroneous clinical consequences of associating risk to the millions of ECGs misread as "early repolarization" on the basis of ST elevation. Furthermore, the application of standardized terminology and computerized ECG technology to future studies investigating abnormalities on the downslope of the R wave would greatly facilitate the translation of laboratory data regarding these phenomena to clinical practice. ${ }^{18-21}$ The first 
step would be to reserve the term early repolarization for ST elevation (with or without J waves or slurring), as first reported- ${ }^{21}$ and to adopt new titles for phenomena on the downslope of the $\mathrm{R}$ wave.

The primary limitation of our study is that, compared with the general population, our population included a larger representation of men. Given that resting ER is more prevalent in men, however, our selection increased the power of detecting differences between ER patterns. Nonetheless, this makes our study not generalizable to women with ER. Furthermore, the ECGs were not ordered per protocol but for various clinical indications according to the discretion of the ambulatory practitioners.

\section{CONCLUSION}

Common patterns of ER without concomitant $Q$ waves or TWI are not associated with increased risk of cardiovascular death. Our results confirm the clinical impression and traditional dogma that classic ER based on ST elevation is a benign entity. The novel combination of $Q$ waves or TWI and ER, however, is associated with increased cardiovascular death.

\section{ACKNOWLEDGMENTS}

The opinions expressed in this article do not necessarily represent the views or policies of the Department of Veterans Affairs.

Abbreviations and Acronyms: $\mathrm{CV}=$ cardiovascular; ECG = electrocardiogram; ER = early repolarization; $\mathrm{HR}=$ hazard ratio; $\mathbf{T W I}=$ T-wave inversion; $\mathbf{V A}=$ Veterans $\mathrm{Ad}$ ministration

Potential Competing Interests: Dr Froelicher is co-owner and Chief Medical Officer of Cardea Associates, Inc, Woodinville, WA, developers of software and hardware for screening for cardiovascular risk.

Correspondence: Address to Karim Sallam, MD, Division of Cardiovascular Medicine, Falk CVRC, Stanford University, 300 Pasteur Dr, Stanford, CA 94305 (sallam@ stanford.edu).

\section{REFERENCES}

1. Haissaguerre M, Derval N, Sacher F, et al. Sudden cardiac arrest associated with early repolarization. N Engl J Med. 2008; 358(19):2016-2023.

2. Tikkanen JT, Anttonen $O$, Junttila MJ, et al. Long-term outcome associated with early repolarization on electrocardiography [published online ahead of print November 16, 2009]. N Engl J Med. 2009;36I (26):2529-2537.

3. Klatsky AL, Oehm R, Cooper RA, Udaltsova N, Armstrong MA. The early repolarization normal variant electrocardiogram: correlates and consequences. Am J Med. 2003; I 5(3): 171-177.

4. Uberoi A, Jain NA, Perez M, et al. Early repolarization in an ambulatory clinical population [published online ahead of print October 10, 201 I]. Circulation. 201 1;124(20):2208-2214.
5. Blackburn $\mathrm{H}$. Classification of the electrocardiogram for population studies: Minnesota code. J Electrocardiol. 1969;2(3):305310.

6. Sinner MF, Reinhard W, Müller M, et al. Association of early repolarization pattern on ECG with risk of cardiac and allcause mortality: a population-based prospective cohort study (MONICA/KORA). PLoS Med. 20I 0;7(7):el0003।4.

7. Haruta D, Matsuo $K$, Tsuneto A, et al. Incidence and prognostic value of early repolarization pattern in the 12 -lead electrocardiogram [published online ahead of print June 6, 201 I]. Circulation. 201 1;123(25):2931-2937.

8. Tikkanen JT, Junttila MJ, Anttonen $O$, et al. Electrocardiographic phenotypes associated with favorable long-term outcome [published online ahead of print May 31, 201 I]. CircuIation. 201 1:123(23):2666-2673.

9. Rosso R, Glikson E, Belhassen B, et al. Distinguishing "benign' from "malignant early repolarization": the value of the STsegment morphology [published online ahead of print September 10, 201 I]. Heart Rhythm. 2012;9(2):225-229.

10. Perez MV, Friday K, Froelicher V. Semantic confusion: the case of early repolarization and the J point [published online ahead of print February 14, 20I2]. Am J Med. In press.

11. Mortara-Instruments. Physicians Guide Veritas 12 Lead Interpretative Program 2005. www.documark.com/Documents/ng/ 9515-00 I-60.pdf. Accessed August 5, 201 I.

12. Philips. 12 Lead ECG Algorithm Physicians Guide 2005. http:// incenter.medical.philips.com/doclib/enc/fetch/2000/4504/577243/ 577245/577817/5778/8/12-Lead Algorithm Physicians Guide for Algorithm Version PH080A\%2c (ENG).pdf\%3fnodeid\% 3d3325283\%26vernum\%3dl. Accessed August 5, 2011.

13. WelchAllyn. Physician's Guide. 2009. http://www.welchallyn. com/documents/Cardiopulmonary/Electrocardiographs/CP300/ PhysicianGuide_200909 I6_CP300.pdf. Accessed August 5, 2011.

14. GE-Healthcare. Diagnostic ECG Algorithms. 2010. http:// www.gehealthcare.com/euen/cardiology/products/diagnostic_ ecg/algorithms/index.html. Accessed August 5, 201 l.

15. Tsai T, Fridsma DB, Gatti G. Computer decision support as a source of interpretation error: the case of electrocardiograms. J Am Med Inform Assoc. 2003; 10(5):478-483.

16. Southern WN, Arnsten JH. The effect of erroneous computer interpretation of ECGs on resident decision making [published online ahead of print May 21, 2009]. Med Decis Making. 2009;29(3):372-376.

17. Brailer DJ, Kroch E, Pauly MV. The impact of computerassisted test interpretation on physician decision making: the case of electrocardiograms. Med Decis Making, 1997;17(1): 80-86.

18. Boineau JP. The early repolarization variant: normal or a marker of heart disease in certain subjects. J Electrocardiol. 2007;40(I):3.ell-3.el6

19. Antzelevitch C, Yan GX. J wave syndromes [published online ahead of print December II, 2009]. Heart Rhythm. 2010;7(4): 549-558.

20. Nattel S. Sudden cardio arrest: when normal ECG variants turn lethal. Nat Med. 20 I0; 16(6):646-647.

21. Grant RP, Estes EH Jr, Doyle JT. Spatial vector electrocardiography: the clinical characteristics of S-T and T vectors. Circulation. 1951;3(2): 182-197. 